\title{
Correction to: Metabolic Profile of 3-Acetyl-11-Keto- $\beta$-Boswellic Acid and 11-Keto- $\beta$-Boswellic Acid in Human Preparations In Vitro, Species Differences, and Bioactivity Variation
}

Yonglei Cui, ${ }^{1}$ Xiangge Tian, ${ }^{2}$ Jing Ning, ${ }^{1}$ Chao Wang, ${ }^{1,3}$ Zhenlong Yu, $^{1}$ Yan Wang, ${ }^{1}$ Xiaokui Huo, ${ }^{1}$ Lingling Jin, ${ }^{1}$ Sa Deng, ${ }^{1}$ Baojing Zhang, ${ }^{1}$ and Xiaochi $\mathrm{Ma}^{1,3}$

published online 14 August, 2019

Correction to: AAPS J.

https://doi.org/10.1208/s12248-016-9945-7

The authors regret that Fig. 7d for the group: LPS+M-2 $(80 \mu \mathrm{M})$ was mistakenly uploaded. The correct microscopy image of Fig. $7 d$ for LPS+M-2 $(80 \mu \mathrm{M})$ group is given below. The corrections do not affect any of the results or conclusions as presented in the original publication. We apologize for any inconvenience.

Original: Fig. 7d LPS+M-2 (80 $\mu$ M)
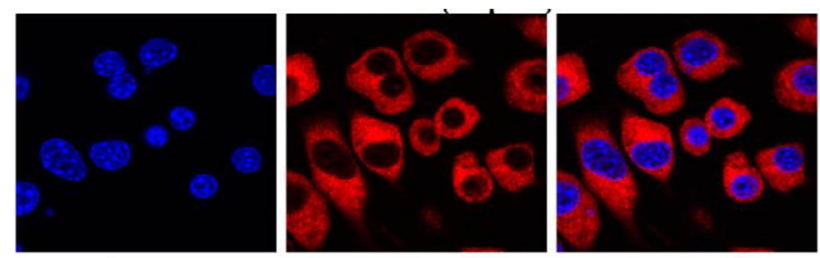

LPS+M-2 (80 $\mu$ M)
Correction:
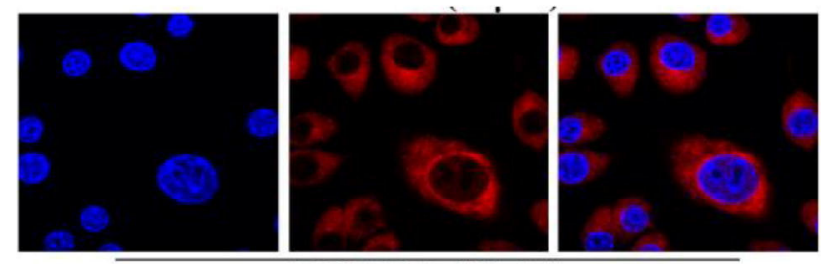

LPS+M-2 (80 $\mu \mathrm{M})$

Publisher's Note Springer Nature remains neutral with regard to jurisdictional claims in published maps and institutional affiliations.

Yonglei Cui and Xiangge Tian contributed equally to this work.

The online version of the original article can be found at https:// doi.org/10.1208/s12248-016-9945-7

\footnotetext{
${ }^{1}$ College of Pharmacy, Academy of Integrative Medicine, Dalian Medical University, 9 West Section, Lvshun South Road, Lvshunkou District, Dalian, 116044, China.

${ }^{2}$ College of Basic Medical Science, Dalian Medical University, Dalian, China.

${ }^{3}$ To whom correspondence should be addressed. (e-mail: wach_edu@sina.com; maxc1978@163.com)
} 